\title{
Using the Shape Characteristics of Rain to Identify and Remove Rain from Video
}

\author{
Nathan Brewer ${ }^{1,2}$ and Nianjun Liu ${ }^{1,2}$ \\ ${ }^{1}$ RSISE, The Australian National University \\ nathan.brewer@rsise.anu.edu.au \\ ${ }^{2}$ NICTA Canberra Lab \\ nianjun.liu@nicta.com.au
}

\begin{abstract}
Dynamic weather effects such as rain cause rapid, distracting motion in a video sequence. This paper aims to remove rain and similar effects from video footage using a multi-step approach; Regions are identified as being potentially affected by rain if they exhibit a shortduration intensity spike. Falling rain drops are imaged by a video camera in a predictable way, as a streak with a consistent range of possible aspect ratios. To preserve scene motion, regions identified by this criterion are investigated, and those that do not fit into the expected range of aspect ratios are ignored. Information about the direction of rainfall is also used to reduce false detections. The effectiveness of this technique is shown on a number of video sequences. The method presented provides advantages over existing techniques.
\end{abstract}

\section{Introduction}

As outdoor surveillance becomes increasingly prevalent in society, vision algorithms that can handle the complex and unpredictable behaviour caused by changing light conditions and weather effects are becoming more important. This paper aims to detect and reduce the visibility of dynamic weather, specifically rain from video sequences. Rain has a negative effect on the performance of other vision algorithms, and can be distracting for a human observer.

Reducing or removing the effects of rain while preserving scene information is a difficult task, as rain streaks move very quickly through a scene and are difficult to separate from other motion in a scene, such as that caused by camera motion. Additionally, the visual appearance of rain depends both on the background of the streak and other scene lighting conditions, which makes it difficult to build a general appearance model.

Exploration of the physical and statistical properties of rain have been explored numerous times in the past, largely in an atmospheric science context. In recent times, a number of groups have approached the problem of reducing the appearance of rain from video in a variety of different ways.

Early methods for reducing the visibility of dynamic weather effects used a temporal median filter for each pixel [12. While this approach was successful at reducing the effects of rain and snow in the video sequence, this type of filter 
resulted in blurring artifacts in footage with a moving camera or scene motion. More recent work 3415] utilises additional properties of rain to identify and suppress only rain streaks, reducing the number of blurring artifacts generated.

Garg and Nayar [4] present a method that uses the optical properties of a falling rain drop to segment a video scene into rain and non-rain regions. Zhang et al [5] make use of the temporal property of rain, that any given pixel is not always covered by rain, together with the chromatic property of rain, that a rain drop will affect the red, green and blue channels of a pixel in a predictable fashion to improve segmentation. Finally, Barnum et al [3] use a global appearance model to identify the typical frequency-domain behavior of rain and snow and present a frequency-domain filter to reduce its visibility.

Each of these methods uses a significant number of frames in order to function optimally. The correlation filter presented by Garg and Nayar [4] uses 30 frames to identify rain-affected regions. The temporal property of Zhang et al [5] is applied across the entire video sequence. Finally, the spatio-temporal frequency method of Barnum et al 3] requires $2 N-1$ frames, where $\mathrm{N}$ is the number of desired removal cycles. This dependence on multiple frames makes these types of algorithms difficult to apply to short-duration sequences with rain or to apply in a short-delay online process.

The approach outlined in this paper uses only the local frame neighborhood to identify and remove the effects of rain based on both the optical and physical properties of a rain streak. In this way, we present a system that is able to reduce the visual effects of rain using fewer frames than similar techniques.

Section two of this paper outlines the algorithm that has been developed to reduce the appearance of rain. In section three we present qualitative results of the algorithm on a number of video sequences and a discussion of the effectiveness of the algorithm, and section four provides a brief summary and introduces potential future work.

\section{Identifying and Removing Streaks}

In this section, we describe the visual appearance of a typical rain streak as imaged by a video camera and a method for identifying and removing such streaks while preserving as much scene information as possible. We do this in a three step process, with an optional fourth step. The algorithm involves first identifying candidate patches, then eliminating those that do not meet the shape properties of rain. If the scene allows, we then identify the likely direction of rainfall to further eliminate false detections. Finally, we replace remaining candidate pixels with an approximation of their background.

\subsection{Identification of Potential Rain-Affected Regions in Video}

To isolate regions in a video which may contain rain, we can make use of Garg and Nayar's 4 photometric model to identify pixels which have potentially been affected by rain. If we identify pixels which undergo a short, positive intensity 
spike, we can build a set of candidate pixels corresponding to pixels that have potentially been affected by rain. As we expect the spike to be short duration, we need only compare the current frame to the previous and following frames. Given that real video is noisy, we select candidate pixels which exhibit a gray level that is higher by at least some threshold in the current frame than in the previous and following frames.

A threshold value of 3 gray levels was found to produce good results. This method allows for intensity changes in the background, provided that the rain streak is brighter than the background in both the previous and following frames. If the same pixel is affected by rain in more than one consecutive frame, this criterion fails to identify a pixel as being affected by rain. In practice, this is rarely a problem, as the velocity of a raindrop is high enough that it is only observable in a given pixel for a single frame, and the probability of different drops being present in the same pixel in consecutive frames is quite low.

In order to investigate the physical properties of each individual streak, contiguous blocks of candidate rain pixels are identified and labelled. As the presence of noise can cause pixels within a streak to be misclassified, a simple morphological fill operation is performed. To reduce noise, all identified regions with fewer than a small threshold $Y$ pixels are removed from the set of candidate rain pixels. A $Y$ value of 5 was experimentally found to be effective.

\subsection{Shape Properties of a Raindrop}

The terminal velocity of a raindrop depends on its diameter, and Foote and DuToit 6] developed an equation which describes the approximate velocity of a raindrop in metres per second as:

$$
v(d)=-0.2+5.0 d-0.9 d^{2}+0.1 d^{3}
$$

where $\mathrm{d}$ is the drop diameter in millimetres.

Barnum et al 3 described the length $l$ and breadth $b$ of a rain streak in terms of the diameter of the drop, its distance from the camera $z$ and various camera properties as:

$$
\begin{aligned}
& b(d, z)=\frac{d f}{z} \\
& l(d, z)=\frac{v(d) e f}{z}+\frac{d f}{z}
\end{aligned}
$$

where $f$ is the focal length of the camera and $e$ is the exposure time.

In order to identify rain streaks in video, we can exploit these parameters. If we define the minimum and maximum sizes for a raindrop visible to a camera in the same fashion as 3 at $0.1 \mathrm{~mm}$ and $3 \mathrm{~mm}$ respectively, we can calculate a range of aspect ratios which correspond to those of visible rain streaks.

$$
\begin{aligned}
A R(d) & =\frac{l(d, z)}{b(d, z)} \\
& =\frac{v(d) e}{d}+1
\end{aligned}
$$


We can see that the aspect ratio of a rain streak depends only on the diameter of the raindrop and the exposure time of the camera. As expected, this shows that a shorter exposure time on the camera will result in a less stretched visible streak regardless of the distance of a drop from the camera or other camera parameters.

Using an exposure time of $16 \mathrm{~ms}$, we then get an aspect ratio range of $47.58 \leq$ $A R \leq 51.13$ for a drop of water. If the exposure time of the camera is unknown, we can assume that it lies in the range of a typical consumer video camera, between 1 and 40ms. Given this, for an unknown camera we have a range of possible rain streak aspect ratios of 3.9 to 95 . Using such a large range of values, however, results in less effective separation of rain and non-rain regions than when the exposure time is known.

Calculating the aspect ratio of a streak in an image is quite challenging, as they are often deformed and, in most rain storms, the outline of a rain streak is difficult to prcisely determine. Because of this, instead of working directly with identified streaks, we find the ellipse with the equivalent second moments as the extracted blob. Assuming that the blob identified is entirely due to a single rain streak, this ellipse will have the properties expected of a falling rain drop. The aspect ratio of such an ellipse is taken as the major axis length divided by the minor axis length.

\subsection{Finding the Direction of Rainfall}

If a video sequence contains only small amounts of non-rain motion, we can also calculate a direction of rainfall based on the orientation of identified streaks, which can help to eliminate candidate blobs that are not due to rain.

We can easily find the orientation of the ellipses identified as having an appropriate aspect ratio in the previous section, and thus the orientation of the blob they are approximating. From this, we construct a histogram of streak orientation, with bins $5^{\circ}$ wide. Assuming that rain falls in an approximately constant direction within any given frame, we can assume that all blobs with orientations that occur in bins with a low proportion of the total scene motion are not rain. Experimentally, we found that discarding bins with less than $10 \%$ of the total was effective.

This additional step further reduces the amount of non-rain scene motion that is erroneously removed, although it is only applicable in sequences with a high volume of rain motion.

\subsection{Correcting Rain-Occluded Streaks}

In order to remove the visual effects of a rain streak, we want to replace the information in each pixel in a particular streak with an estimate of what the pixel information would have been had it not been raining. As the identification of a rain streak relies on a relatively constant background, we can substitute the mean of the pixel values at the previous and following frame for each pixel identified as being affected by rain: 


$$
P_{t}=\frac{P_{t-1}+P_{t+1}}{2}
$$

As the edges of identified blobs can be noisy, a morphological closing is performed to improve the visual output.

\subsection{Summary of Rain Removal Procedure}

In order to accurately identify and reduce the visible effects of rain in a video sequence, we apply a relatively simple algorithm. First, we identify which pixels may have been affected by rain by locating regions which display a short-duration intensity spike. We then investigate each blob of candidate pixels by observing its shape and orientation characteristics, discarding those that do not meet aspect ratio or orientation conditions, described above. Finally, for pixels that are identified as rain, we replace the affected pixel with the average of the pixel value in the previous and following frames.

\section{Experimental Results and Discussion}

The rain identification and removal method presented in section 2 was applied to a number of sequences featuring rain that have been used in previous work on rain removal. The algorithm performs reasonably well, removing a significant proportion of rain from a sequence with minimal distortion of scene information, as shown in Figure1. While some rain streaks are missed, a significant proportion of rain has been identified and removed without camera stabilisation and with only three consecutive frames required.

Due to the difficulty of identifying and hand-labelling rain streaks, we were unable to develop a quantitative method for determining rain removal quality for all images. Presented in Figure 2 is a $100 \times 100$ pixel region of Figure 1 in which we hand-marked all pixels determined to be affected by rain.

Initial segmentation of the video is the major cause of misclassified rain streaks. Typically, misclassifications occur when multiple rain streaks intersect at some point, generating an unusual shape which does not meet the aspect ratio

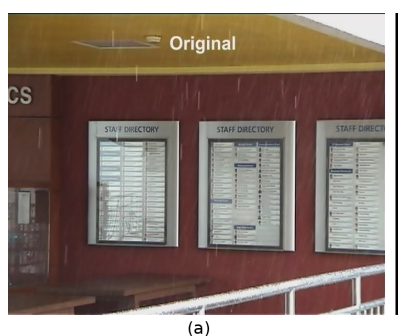

(a)

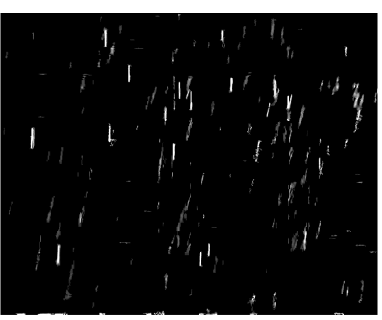

(b)

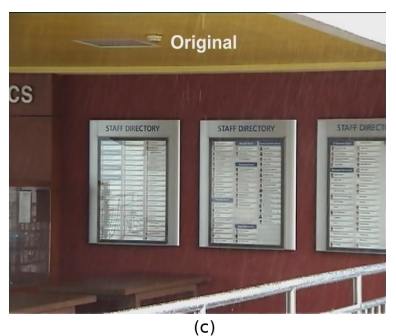

(c)

Fig. 1. A frame from a scene with a shaking camera and falling rain, from [5]. (a) is the original image, (b) the identified rain streaks and (c) the image after rain removal. 


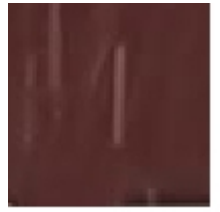

(a)

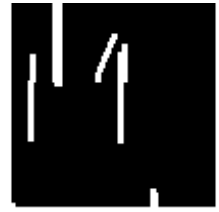

(b)

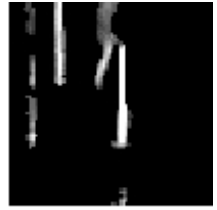

(c)

Fig. 2. A zoomed in section of Figure 1 (a) showing pixels identified as rain by hand (b) and by the proposed algorithm (c)

criteria. The other major cause of misclassifications occur when a rain streak intersects some other scene motion. Severely out of focus or distant rain streaks are difficult to detect using the intensity spike criterion. Additionally, water streaks which are caused by dripping water that is not rain can often have different orientations or sizes that do not correspond to the properties expected of rain.

In videos in which the direction of rain was seen as constant within a frame, and rain motion was the primary cause of initial detection, the direction of rainfall was taken into account. This further increased the accuracy of classification, as shown in Figure 3. As can be clearly seen, the streaks corresponding to the man's shirt that are changed in Figure 3 (b) are not changed when the direction parameter is applied, as they are not oriented in the primary rain direction.

In practice, particularly in sequences taken from movies, the aspect ratio of some raindrops does not fall in the expected range. This is most likely because much rain in movies is created using a rain tower, and so has not reached terminal velocity which results in a shorter streak. As such, the aspect ratio range for identifying a streak had to be broadened to identify these artificial rain drops.

This model can be applied in addition to other methods to reduce false detections. For example, when combined with the rain removal algorithm presented by Garg and Nayar in [4] as an additional step following the segmentation of rain regions. Doing so allows for identification and removal of blurring artifacts that appear as scene motion intersects with an area identified as being rain. Figure 4

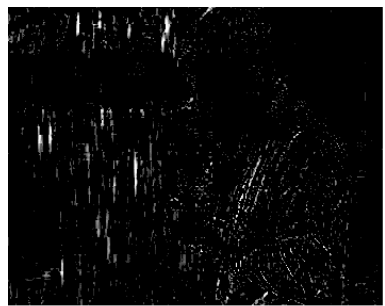

(a)

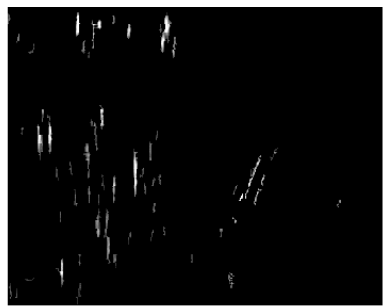

(b)

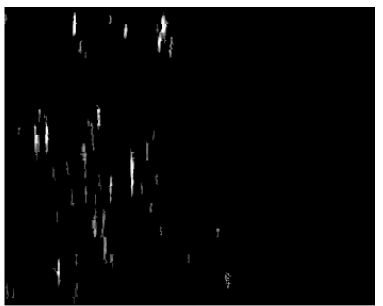

(c)

Fig. 3. A difference image from a frame from the film Magnolia showing pixels changed by the algorithm in white. (a) shows all potential pixels, (b) those identified by the shape property and (c) those identified by both the shape and direction properties. 


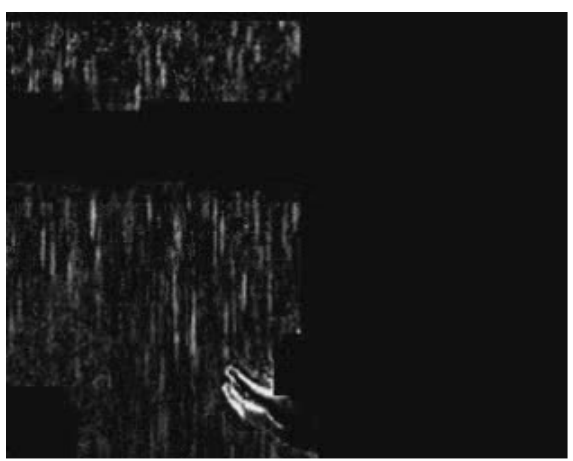

(a)

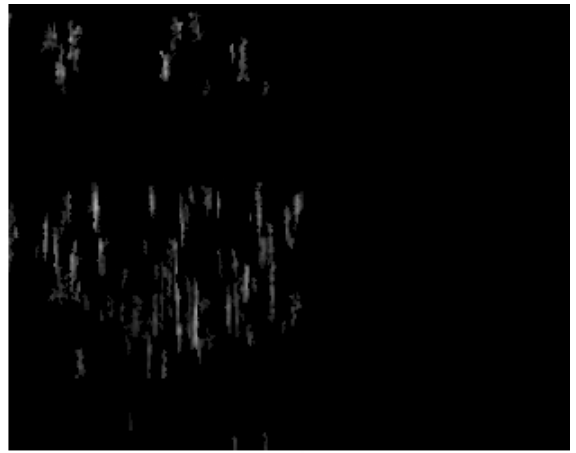

(b)

Fig. 4. A frame from the film Magnolia. (a) shows the pixels identified as rain by Garg and Nayar's method from [4, and (b) shows the streaks that are still identified after application of the presented algorithm.

shows rain identified in a frame from Magnolia using the method presented in 4 and the streaks that are identified as rain by the presented algorithm. As can clearly be seen, the detection that results from the man moving his hand is no longer identified as a rain streak in 4(b), resulting in significantly less blurring of scene motion.

Non streak-like background rain is not removed by this algorithm, as it often does not meet the segmentation criteria. However, the clear, bright foreground rain streaks which cause the majority of distraction to a human observer are detected and removed by the algorithm. This significantly improves the visual appearance of rain affected video sequences.

\section{Conclusions and Future Work}

This algorithm provides a method for reducing the amount of rain present in a section of video footage while preserving scene motion and background textures. By focusing on the physical properties of a rain streak, we are able to separate intensity changes due to rain from those due to scene motion. Other approaches require more frames to identify and remove rain from a video than the approach illustrated here, resulting in longer unavoidable lag times. The algorithm is able to be easily adapted to different scene types and camera parameters.

In order to further improve the algorithm, background subtraction methods may be useful to help isolate and segment rain streaks and scene motion, which can then be used to augment the intensity spike criterion and provide a better segmentation. Stabilisation techniques may also be useful to reduce camera motion and improve segmentation. Camera stabilisation techniques may also improve initial segmentation. 
In general, isolated rain streaks over a relatively stable background can be readily identified as such and removed from a video sequence with minimal disruption of scene motion using only three frames with the algorithm presented.

\section{References}

1. Hase, H., Miyake, K., Yoneda, M.: Real-time snowfall noise elimination. In: Proceedings of 1999 International Conference on Image Processing, ICIP 1999, vol. 2, pp. 406-409 (1999)

2. Starik, S., Werman, M.: Simulation of rain in videos. In: Texture Workshop, ICCV, vol. 2, pp. 406-409 (2003)

3. Barnum, P., Kanade, T., Narasimhan, S.: Spatio-temporal frequency analysis for removing rain and snow from videos. In: Workshop on Photometric Analysis For Computer Vision (2007)

4. Garg, K., Nayar, S.: Vision and rain. International Journal of Computer Vision 75, 3-27 (2007)

5. Zhang, X., Li, H., Qi, Y., Leow, W.K., Ng, T.K.: Rain removal in video by combining temporal and chromatic properties. In: IEEE International Conference on Multimedia and Expo 2006, pp. 461-464 (July 2006)

6. Foote, G.B., Du Toit, P.S.: Terminal Velocity of Raindrops Aloft. Journal of Applied Meteorology 8, 249-253 (1969) 